\section{Ein Satz heiße Ohren}

— in vierjähriger marokkanischer Jun- ge wurde unter dem Verdacht einer in diesem Alter seltenen beidseitigen Perichondritis der Ohren überwiesen. Beide Ohrmuscheln waren geschwollen und gerötet, wobei die Rötung scharf zum Ohrläppchen hin begrenzt war. Einschlägige Blutuntersuchungen wie BKS und auf Autoantikörper bestätigten den Verdacht auf eine Perichondritis allerdings nicht. Die Mutter des Jungen erwähnte zufällig, dass vor kurzem in der Gegend eine schreckliche Mückenplage geherrscht habe. Als man den Jungen daraufhin näher untersuchte, fanden sich an Armen und Beinen zahlreiche Mückenstiche. Daraufhin änderte man die Verdachtsdiagnose in
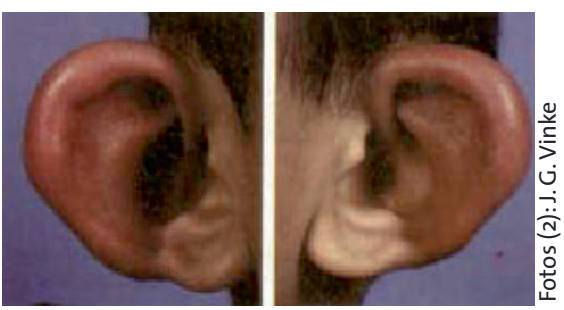

eine Hypersensitivitätsreaktion gegen zufälligerweise mehrere gleichzeitig erfolgte Mückenstiche in beide Ohrmuscheln. Wieder einmal ein Beweis dafür, dass man sich selbst durch eine offensichtliche Blickdiagnose nicht davon abhalten lassen sollte, in üblicher Weise eine komplette körperliche Untersuchung durchzuführen.

hsf

Vinke JG et al. Lancet 2004; 363: 1421

\title{
Reha im Rückgang
}

_ rstmals seit 1997 gab es im Jahr 2002 weniger Patienten in Vorsorge- oder Rehabilitationseinrichtungen. Die Zahl der Behandlungsfälle lag mit 2,0 Millionen um 2,7\% niedriger als im Jahr 2001. Auch die Anzahl der Betten und Einrichtungen ging zurück: 2002 standen mit 185.000 Betten $2,4 \%$ weniger als im Vorjahr in den Vorsorge- oder Rehabilitationseinrichtungen, deren Zahl insgesamt um 45 Einrichtungen abnahm. Somit setzt sich der seit Ende der 90er Jahre anhaltende Einrichtungs- und Bettenabbau weiter fort.

Statistisches Bundesamt, 10. November 2004

\section{Kommt Leukämie mit Allergie?}

öntgenkontrastmittel (RKM) sind trijodierte Benzole, die vor allem wegen der hochdosierten und raschen Gabe zu Unverträglichkeitsreaktionen führen können. Neben toxischen Reaktionen sind Überempfindlichkeitsreaktionen vom Soforttyp, aber auch Spättypreaktionen bekannt. Dr. Knut Brockow aus München empfiehlt, vorsorglich ionische RKM zu meiden. Allerdings gibt es für die neueren nichtionischen RKM noch keine evidenzbasierte Prämedikationsempfehlung. Eine Task Force „Rönt-
genkontrastmittel-Unverträglichkeit“ des European Network of Drug Allergy (ENDA) trägt derzeit den aktuellen Kenntnisstand zusammen. Eine Studie der Task Force wird die Möglichkeit diagnostischer Tests und Pathomechanismen der Sofort- und Spätreaktionen auf RKM untersuchen.

FK

Brockow K. 20. Fortbildungskongress „Fortschritte der Allergologie, Immunologie und Dermatologie“, Davos, 1.-4. September 2004

\section{Lunge im Mittagstief}

D ie Lungenkapazität unterliegt einer ausgeprägten zirkadianen Rhythmik. Untersuchungen an 4.800 Patienten des Long Island Jewish Medical Center in New York haben zusätzlich zu dem bekannten Tief in den frühen Morgenstunden auch mittags eine merkliche Reduktion der Lungenfunktion offenbart. Zwischen dem Tief am Mittag und dem Tageshoch am späten Nachmittag beträgt die Differenz der Lungenfunktion zwischen 15 und 20\%. Dies sollte bei der Behandlung von Lungenerkrankungen berücksichtigt werden, empfiehlt Studienleiter Dr. Boris Medarov: Möglicherweise könnte durch eine zeitgenaue Einnahme der Medikamentenverbrauch bei Asthmatikern gesenkt werden, und Physiotherapie sollte möglichst am späten Nachmittag eingesetzt werden. Auch könnte es sinnvoll sein, die Extubation bei beatmeten Patienten auf diesen Zeitpunkt zu legen, weil dann die Chancen der Spontanatmung am besten sind.

bmm

New Scientist, 26. Oktober 2004

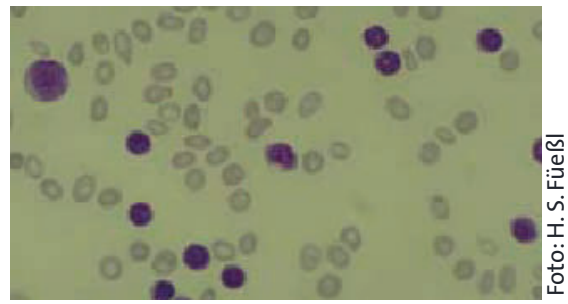

D ie Frage, ob es einen Zusammenhang zwischen allergischen und hämatologischen Erkrankungen gibt, wird seit Jahren kontrovers diskutiert. Eine große Kohortenstudie, bei der die Lebensdaten von 16.500 Schweden über 31 Jahre hinweg ausgewertet wurden, fand jetzt positive Korrelationen: Personen mit Asthma bronchiale oder chronischer Urtikaria hatten ein rund doppelt so hohes Risiko, in ihrem Leben auch an Leukämie zu erkranken (relatives Risiko 1,6 bzw. 2,1). Neurodermitis in der Kindheit hatte eine relatives Risiko von 2,3 zur Folge, ein NonHodgkin-Lymphom zu entwickeln. Für Heuschnupfenpatienten konnte dagegen kein Zusammenhang mit Leukämie oder Lymphomen gefunden werden. es

Soderberg KC et al. BMC Public Health 2004; 4: 51 